\title{
Tetranychus urticae (Koch, 1836) em cultivo de lúpulo Humulus lupulus L. (Cannabaceae) no Rio Grande do Sul, Brasil - primeiro relato de ocorrência
}

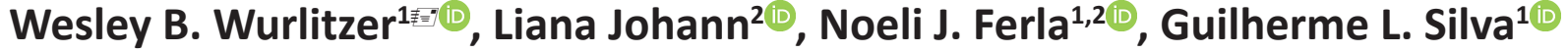 \\ ${ }^{1}$ Laboratório de Acarologia, Tecnovates, Universidade do Vale do Taquari - Univates, Lajeado, Rio Grande do Sul, Brasil. ${ }^{2}$ CNPq \\ Researcher.
}

莑="Corresponding author: wesleeywurlitzer@gmail.com

Edited by: Samuel Roggia

Received: June 15, 2020. Accepted: June 29, 2020. Published: August 31, 2021.

Tetranychus urticae (Koch, 1836) in Humulus lupulus L. (Cannabaceae) hop cultivation in Rio Grande do Sul, Brasil - first report of occurrence

Abstract. Tetranychus urticae Koch (Koch, 1836) is considered one of the main pests in hop crops Humulus lupulus L. (Cannabaceae) when found in high densities, as it causes leaf browning, plant defoliation and attack on flowers, reducing productivity and quality of the plant production. In this sense, the present study aims to record for the first time the occurrence of T. urticae and its injury to hops in Rio Grande do Sul State. The injuries observed were irregular spots, web formation and leaf browning. It was also observed Phytoseiulus macropilis (Banks, 1904) preying on T. urticae.

Keywords: Phytoseiidae, phytophagous, predator, Tetranychidae.

O lúpulo Humulus lupulus L. (Cannabaceae) é cultivado principalmente nos Estados Unidos e na Alemanha, encontrandose em expansão no Brasil. A espécie é caracterizada principalmente por suas flores denominadas de cones geradas apenas pelas plantas fêmeas (Teghtmeyer 2018; Spósito et al. 2019). Os cones apresentam glândulas de lupulina que produzem compostos utilizados na produção de fármacos e cervejas. Existe diferentes cultivares de lúpulos, como os de amargor, aroma e dupla aptidão (Aghamiri 2016; Dodds 2017; Bocquet 2018; Rettberg 2018; Spósito et al. 2019).

Nos países produtores, um dos principais problemas enfrentados no cultivo está relacionado à ocorrência do ácaro fitófago Tetranychus urticae Koch (Koch, 1836) (Acari: Tetranychidae; two spotted spider mite - TSSM) (Aghamiri 2016; Dodds 2017; Bocquet 2018; Rettberg 2018; Spósito et al. 2019). TSSM está associado a diversas culturas, como morango, feijão, tomate e lúpulo (Gallo et al. 2002; Nascimento et al. 2019). Ele utiliza seus estiletes para perfurar a epiderme das folhas e sugar o conteúdo celular (Moraes \& Flechtmann 2008; Gent et al. 2009). No lúpulo, quando encontrado em altas densidades, ocasiona manchas irregulares, acarretando bronzeamento e desfolhamento. Além disso, é responsável por perdas ao se alimentar dos cones, causando descoloração, ressecamento e tornando-os quebradiços, podendo acarretar a perda total da produção (Gent et al. 2009; Woods et al. 2021). No Brasil, são poucos os estudos sobre pragas e doenças relacionadas ao cultivo do lúpulo, devido à sua recente introdução no país (Spósito et al. 2019). A presença do TSSM nesta cultura foi registrada até o momento, apenas para os estados de São Paulo e Rio de Janeiro (Aquino et al. 2019; Nascimento et al. 2019; Teixeira \& Silva 2019).

As principais estratégias de manejo de TSSM utilizam o controle químico ou biológico (Gent et al. 2009; Vostrel 2010; Dodds 2017). Até o momento não há produtos químicos ou biológicos registrados no Ministério da Agricultura, Pecuária e Abastecimento, do Brasil, para o controle de ácaros em lúpulo. Pelo fato de os acaricidas terem demonstrado efeitos negativos promovendo a resistência, o uso de ácaros predadores da família Phytoseiidae tem sido o principal meio para o controle nos maiores países produtores (Strong \& Croft 1993, 1995; Gent et al. 2009; Dodds 2017). Até o momento, as principais espécies estudadas e reportadas como potenciais controladoras foram: Galendromus occidentalis (Nesbitt, 1951), Neoseiulus fallacis (Garman, 1948), Typhlodromus pyri Scheuten, 1857, Amblyseius andersoni (Chant, 1957) e Phytoseiulus persimilis Athias-Henriot, 1957 (Lilley \& Campbell 1999; Gent et al. 2009; Dodds 2017).

Neste sentido, o presente estudo teve como objetivo realizar levantamento de ácaros associados ao cultivo comercial de lúpulo no estado do Rio Grande do Sul.

A ocorrência de altas populações de TSSM e suas injúrias foram observadas por agricultores nos municípios de Putinga ( $29^{\circ} 00^{\prime} 17,4^{\prime \prime} \mathrm{S}$ $\left.52^{\circ} 09^{\prime} 27,9^{\prime \prime} \mathrm{W}\right)$ e de Forquetinha $\left(29^{\circ} 23^{\prime} 22,6^{\prime \prime} \mathrm{S} 52^{\circ} 04^{\prime} 60,0^{\prime \prime} \mathrm{W}\right)$, no estado do Rio Grande do Sul, Brasil. Em Forquetinha, o cultivo apresentava dois anos de idade, e o TSSM foi registrado nas cultivares Saaz, lúpulos de aroma (Spósito et al. 2019) e em Sorachi Ace, lúpulos de amargor (Sanekata et al. 2018). Já em Putinga, o cultivo apresentava quatro meses de idade e os ácaros foram registrados nas cultivares Comet e Brwes Gold, lúpulos de amargor (Gent et al. 2009), e em Cascade e Hallertau, lúpulo de aroma (Spósito et al. 2019).

As coletadas foram realizadas no dia 05/04/2021 em Putinga, e dia 19/05/2021 em Forquetinha. Foram coletadas aproximadamente 20 folhas de cada cultivo. A amostragem foi realizada de forma direcionada apenas às folhas com a presença de injúrias. Após a coleta, as folhas foram embaladas em sacos plásticos e armazenadas em uma caixa de isopor com Gelox ${ }^{\circledR}$ e levadas até o Laboratório de Acarologia da Universidade do Vale do Taquari - Univates. No laboratório, as folhas foram observadas usando um microscópio estereoscópio binocular Leica S6E. Os ácaros foram coletados das folhas com auxílio de um pincel de ponta fina, montados em lâminas de microscopia em meio Hoyer (Jeppson et al. 1975), e mantidos em uma estufa a 50ㄷ por aproximadamente sete dias, para fixação e clarificação dos espécimes.

A identificação dos espécimes foi realizada com o auxílio de um microscópio óptico de contraste de fases (Zeiss Imager Z2), tendo como referência as chaves de identificação de Baker \& Tuttle (1994) 
e Chant \& McMurtry (2007). Em todas as amostras analisadas a única espécie de ácaro fitófago encontrada foi $T$. urticae. Os sintomas de injúrias observadas foram manchas irregulares, formação de teia e bronzeamento nas folhas das plantas atacadas por este ácaro (Fig. 1AB).

Para controle de TSSM, espécimes de $P$. macropilis foram retirados da criação estoque do Laboratório de Acarologia da Univates e liberados nos cultivos de lúpulo. Passados três dias da sua liberação, novas amostras de folhas foram obtidas, e analisadas em laboratório, onde foi observada a presença de ovos de $P$. macropilis e espécimes de P. macropilis predando TSSM.

Para o controle de TSSM espécies de Phytoseiidae são reconhecidas e já foram estudadas. Em lúpulo anão na Inglaterra, as cultivares First Gold e Herald, P. persimilis Athias-Henriot, 1957 apresentou maior capacidade de predação, quando comparado a Neuseiulus californicus (McGrego, 1954), sugerindo seu uso quando altas populações de TSSM estão presentes (Barber et al 2003). No Brasil, P. macropilis foi eficiente no controle de TSSM em um cultivo de morango (Oliveira et al. 2009). Ferla et al. (2011) corroboram com este resultado, e demonstram que este ácaro apresenta capacidade rápida de reprodução, indicando seu uso por ser uma espécie nativa do Brasil. Portanto, estes estudos sugerem que $P$. macropilis pode apresentar grandes resultados quanto ao seu potencial controlador biológico de TSSM no cultivo do lúpulo gaúcho e brasileiro.

O TSSM está associado a mais de 1000 espécies de plantas (Grbic et al. 2011). Nos principais países produtores de lúpulo, este ácaro tem sido relatado como praga, por suas altas infestações (Gent et al. 2009; Dodds 2017). Até o momento a presença do TSSM nesta cultura foi registrada apenas para os estados de São Paulo e Rio de Janeiro (Aquino et al. 2019; Nascimento et al. 2019; Teixeira \& Silva 2019)

Apesar de haver relato de que o TSSM pode ocasionar a perda total da produção em cultivos de lúpulo, existem poucos estudos científicos mensurando essas perdas (Gent et al. 2009; Sirrine et al. 2010). Weihrauch (2005) concluiu pela primeira vez em seu trabalho realizado na Alemanha no período próximo da colheita do lúpulo, que o TSSM pode demonstrar risco econômico tanto no rendimento, quanto no teor de ácido dos cones, quando o número ultrapassa 90 ácaros por folha. Woods et al. (2021) corroboram com estes dados, pois concluíram em seu estudo, a previsão para os surtos de TSSM nos cones, antes mesmo das plantas colocarem suas flores, com base em dados algorítmicos criados anteriormente a partir de danos eventuais nos primeiros estágios das plantas.

Neste contexto, este estudo relata pela primeira vez a ocorrência de T. urticae em cultivo comercial de lúpulo no Rio Grande do Sul, auxiliando o diagnóstico de plantas atacadas através da caracterização de suas injúrias na planta.

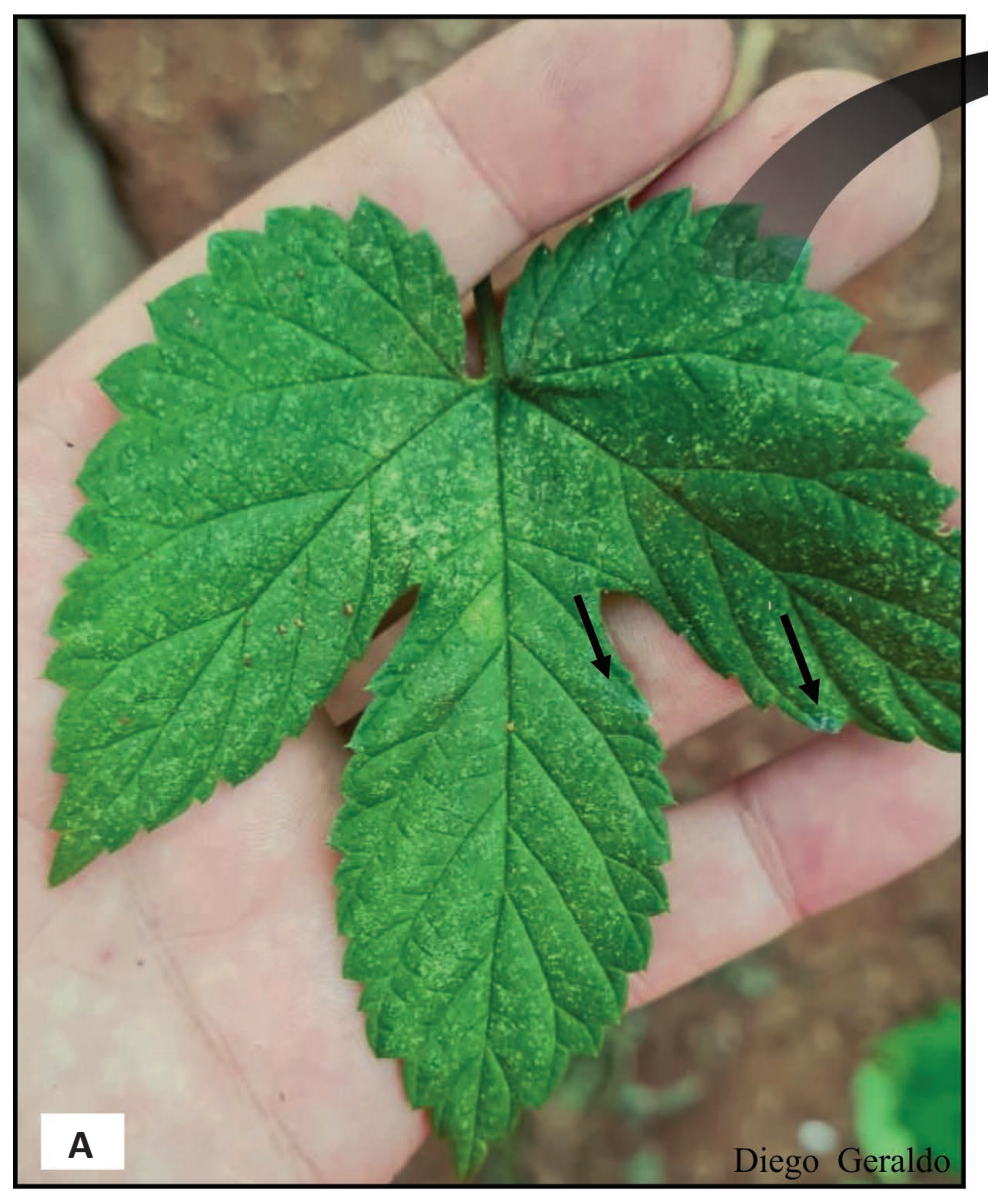

Figura 1. Sintomas observados nas folhas - região adaxial (A) e região abaxial (B).

\section{Agradecimentos}

Os autores agradecem ao Conselho Nacional de Desenvolvimento Científico e Tecnológico (CNPq) pelo apoio financeiro e bolsas de pesquisa (processo PQ no 313658/2020-0), à Universidade do Vale do Taquari - Univates.

\section{Contribuições dos Autores}

WBW conduziu as atividades no laboratório e escreveu o artigo.
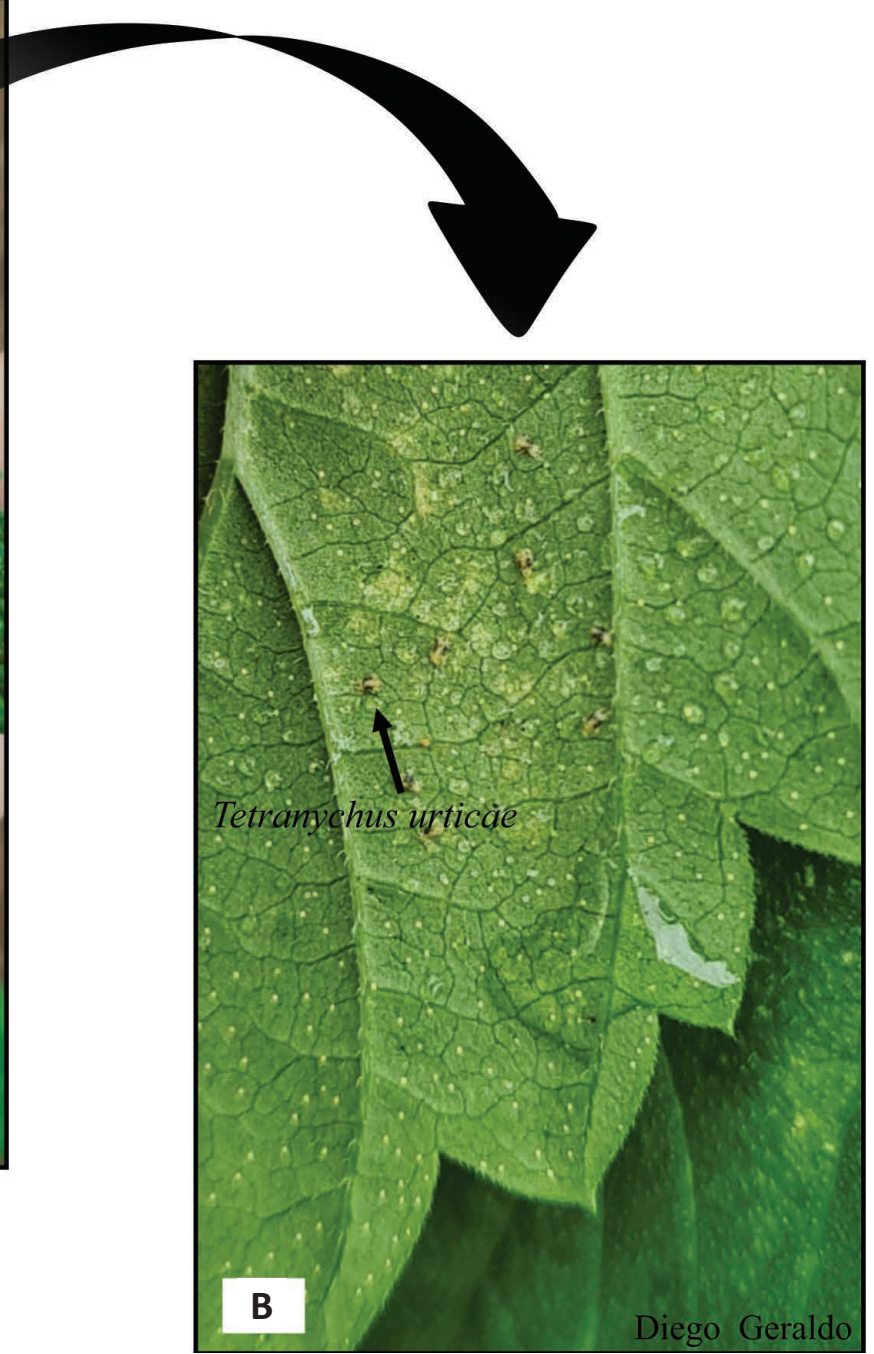

NJF, GLS e LJ contribuíram na redação. GLS obteve financiamento.

\section{Referências}

Aghamiri, V.; Mirghafourvand, M.; Mohammad-Alizadeh-Charandabi, S.; Nazemiyeh, H. (2016) The effect of hop (Humulus lupulus L.) on early menopausal symptoms and hot flashes: A randomized placebo-controlled trial. Complementary Therapies in Clinical Practice, 23: 130-135. doi: 10.1016/j.ctcp.2015.05.001 
Aquino, A. M; Teixeira, A. J.; Assis, A. L. (2019) Referencial Técnico de Atratividade Agropecuária - lúpulo. Nova Friburgo: Embrapa.

Barber, A.; Campbell, C. A. M.; Crane, H.; Lilley, R. (2003) Biocontrol of two-spotted spider mite Tetranychus urticae on dwarf hops by the phytoseiid mites Phytoseiulus persimilis and Neoseiulus californicus. Biocontrol Science and Technology, 13(3): 275-284. doi: 10.1080/0958315031000110300

Baker, E. W.; Tuttle, D. M. (1994) A guide to the spider mites (Tetranychidae) of the United States. West Bloomfield: Indira Publishing House.

Bocquet, L.; Sahpaz, S; Hilbert, J. L.; Rambaud, C.; Rivière, C. (2018) Humulus lupulus $\mathrm{L}$., a very popular beer ingredient and medicinal plant: overview of its phytochemistry, its bioactivity, and its biotechnology. Phytochemistry Reviews, 17(5): 1047-1090. doi: 10.1007/s11101-018-9584-y

Chant, D. A.; McMurtry, J. A. (2007) Illustrated keys and diagnoses for the genera and subgenera of the Phytoseiidae of the world (Acari: Mesostigmata). West Bloomfield: Indira Publishing House.

Dodds, K. A. (2017) Hops: a guide for new growers. New South Wales: Department of Primary Industries.

Ferla, N. J.; Marchetti, M.; Johann, L. (2011) Functional response of Phytoseiulus macropilis under different Tetranychus urticae (Acari: Phytoseiidae, Tetranychidae) population density in laboratory. Zoologia, 28(1): 17-22. doi: 10.1590/S1984-46702011000100003

Gallo, D.; Nakano, O.; Neto, S.S.; Carvalho, R.P.L.; Batista, G.C.; Filho, E.B.; Parra, J.R.P.; Zucchi, R.A.; Alves, S.B.; Vendramim, J.D., et al. (2002) Entomologia Agrícola. Piracicaba: FEALQ.

Gent, D. H.; Barbour, J. D.; Dreves, A. J. (2009) Field Guide for Integrated Pest Management in Hops. Idaho: Oregon State University.

Grbic, M.; Van Leeuwen, T.; Clark, R. M.; Rombauts, S.; Rouzé, P.; Grbic V.; Osvorne, E.J.; Wannes, D.; Ngoc, P.C.T.; Ortego, F., et al. (2011) The genome of Tetranychus urticae reveals herbivorous pest adaptations. Nature, 479: 487-492. doi: 10.1038/nature10640

Oliveira, H.; Fadini, M. A. M.; Venzon, M. (2009) Evaluation of the predatory mite Phytoseiulus macropilis (Acari: Phytoseiidae) as a biological control agent of the two-spotted spider mite on strawberry plants under greenhouse conditions. Experimental and Applied Acarology, 47(4): 275-283.

Jeppson, L. R.; Keifer, H. H.; Baker, E. W. (1975) Mites Injurious to Economic Plants. University of California Press.

Lilley, R.; Campbell, C. A. (1999) Biological, chemical and integrated control of two-spotted spider mite Tetranychus urticae on dwarf hops. Biocontrol Science and Technology, 9(4), 467-473. doi. 10.1080/09583159929433

Moraes, G. J.; Flechtmann, C. H. W. (2008) Manual de acarologia: acarologia básica e ácaros de plantas cultivadas no Brasil. Ribeirão Preto: Holos.

Nascimento, S. R.; Fortuna, G. C.; Avellar Pires, A. B. R. D. (2019) Entomofauna associada ao manejo orgânico e convencional de lúpulo cultivado no oeste paulista. In: I Simpósio Técnico-Científico da Cultura do Lúpulo e II Seminário Técnico da Aprolúpulo. Botucatu: UNESP.

Rettberg, N.; Biendl, M.; Garbe, L. A. (2018) Hop aroma and hoppy beer flavor: chemical backgrounds and analytical tools-a review. Journal of the American Society of Brewing Chemists, 76(1): 1-20. doi: 10.1080/03610470.2017.1402574

Sanekata, A.; Tanigawa, A.; Takoi, K. (2018) Identification and characterization of geranic acid as a unique flavor compound of hops (Humulus lupulus L.) variety Sorachi Ace. Journal of agricultural and food chemistry, 66(46): 12285-12295.

Sirrine, J. R.; Rothwell, N.; Lizotte, E. (2010) Sustainable hop production in the Great Lakes region. Michigan: Michigan State University.

Spósito, M. B.; Ismael, R. V.; Alcântara Barbosa, C. M. D. (2019) A cultura do lúpulo. Piracicaba, SP: Esalq-Divisão de Biblioteca.

Strong, W. B.; Croft, B. A. (1995) Inoculative release of phytoseiid mites (Acarina: Phytoseiidae) into the rapidly expanding canopy of hops for control of Tetranychus urticae (Acarina: Tetranychidae). Environmental Entomology, 24(2): 446-453. doi: 10.1093/ ee/24.2.446
Strong, W. B.; Croft, B. A. (1993) Phytoseiid mites associated with spider mites on hops in the Willamette Valley, Oregon. Journal of the Entomological Society of British Columbia, 90: 45-52.

Teghtmeyer, S. (2018) Hops. Journal of Agricultural \& Food Information, 19(1): 9-20.

Teixeira, A. J.; Silva, M. L. P. (2019) Desenvolvimento e adaptabilidade da cultura do lupulo na região serrana fluminense - perspectivas \& entraves. Niterói: Emater-Rio.

Vostřel, J. (2010) Bifenazate, a prospective acaricide for spider mite (Tetranychus urticae Koch) control in Czech hops. Plant Protection Science, 46(3): 135-138.

Weihrauch, F. (2005) Evaluation of a damage threshold for two-spotted spider mites, Tetranychus urticae Koch (Acari: Tetranychidae), in hop culture. Annals of Applied Biology, 146: 501-509. doi: 10.1111/j.1744-7348.2005.040163.x

Woods, J. L.; Iskra, A. E.; \& Gent, D. H. (2021) Predicting Damage to Hop Cones by Tetranychus urticae (Acari: Tetranychidae). Environmental Entomology, 50(3), 673-684. doi: 10.1093/ee/nvab008 